\section{R. Meyendorf \\ E. Stuber \\ C. Piesbergen \\ B. Reichart \\ E. Kreuzer \\ P. Überfuhr}

A. Welz

\title{
Die Rangfolge psychischer und sozialer Prädiktoren und Kriterien für die Prognose Herztransplantierter - prä- und postoperative Untersuchungen bis zu einem und bis zu drei Jahren nach der Herztransplantation
}

Eingegangen: 2. Januar 1997

Akzeptiert: 16. April 1997
Prof. Dr. R. Meyendorf ( $\triangle$ )

Psychiatrische Klinik der

Ludwig-Maximilians-Universität München

NuBbaumstraße 5

80336 München

\section{E. Stuber}

Meraner Straße 5

85521 Ottobrunn

C. Piesbergen

Psychologisches Institut

der Ludwig-Maximilians-Universität

Leopoldstraße 13

80802 München

B. Reichart - E. Kreuzer $\cdot$ P. Überfuhr

A. Welz

Herzchirurgische Klinik

der Ludwig-Maximilians-Universität

Klinikum Großhadern

Marchioninistraße 15

81377 München
The rank of psychic and social predictors and criteria for prognosis of cardiac transplantation - pre- and postoperative examinations after one year and up to three years after transplantation

Zusammenfassung Bei 44 Herztransplantierten wurden präoperativ und bis zu einem und drei Jahre postoperativ psychiatrisch/psychologische Untersuchungen durchgeführt und psychosoziale Daten erhoben. Die Prädiktoren und Kriterien für eine gute Prognose wurden ihrer Rangfolge nach hierarchisiert. Es ergab sich folgende Prädiktorrangfolge: 1 . die eindeutige Motivation und der unauffällige psychopathologische Befund, 2. der gute soziale Rückhalt und die vor der Transplantation zum Ausdruck gebrachte positive Berufsperspektive, 3. das Bewußtsein der Geborgenheit durch die Familie und die reife psychologische Verarbeitung der Herzerkrankung und der bevorstehenden Herztransplantation, 4. der fortgeschrittene Schweregrad der Herzerkrankung (Schweregrad IV NYHA), 5. der relativ geringe Fernsehkonsum (weniger als an 4 Abenden), und 6. u. a. noch lebende Eltern und das Vorhandensein von Kindern. Für die 7 prognostischen Kriterien ließ sich folgende Kriterienrangfolge ermitteln: 1. die soziale Reintegration, 2. die Lebenszufriedenheit, 3. die Compliance und der psychopathologische Befund, 4. die berufliche Rehabilitation, und 5. die körperliche Belastbarkeit und der körperliche Zustand. Die Studie zeigt, daß es eine unterschiedliche Gewichtung psychosozialer Faktoren vor und nach der Herztransplantation gibt, und daß die soziale Reintegration der beste Indikator für eine erfolgreiche Herztransplantation ist.

Summary 44 patients underwent psychiatric/psychological and psychosocial examinations before and for one to three years after heart transplantation. Predictors and prognostic criteria for a good result were established according to the rank of their significance. The rank sequence for the predictors were 1 . strong motivation and absence of psychiatric symptomatology, 2. good social support and determination to return to work after transplantation, 3. the feeling of being cared for by the family and a mature psychological coping with the heart disease and the expected transplantation, 4. advanced heart failure (stage IV NYHA), 5. watching TV less than 4 evenings a week, 6 . parents still living and having one's own children. The rank sequence for the 7 prognostic criteria for a good result after heart transplantation were 1 . social reintegration, 2. being content with life, 3 . 
good compliance and absence of psychopathology, 4. returning to work, and 5. physical exercise tolerance and general physical condition. The study shows that there is a different magnitude of significance for psychosocial factors before and after heart transplantation and that social reintegration is the best indicator for a good result after heart transplantation.

Schlüsselwörter Herztransplantation, Rangfolge psychiatrischer/ psychosozialer Prädiktoren und Kriterien

Key words Cardiac transplantation, ranking psychiatric/psychosocial predictors and criteria

\section{Einleitung}

Eindeutige Motivation, verläßliche Compliance und ein stabiler sozialer Rückhalt sind auch heute noch - wie bereits in den Anfängen der HT die drei psychosozialen Säulen der Herztransplantation. Als wichtigste Auswahlkriterien sind sie immer auch schon die entscheidenden prognostischen Kriterien. Im Laufe der Zeit haben sich weitere aussagekräftige prognostische Kriterien ermitteln lassen, darunter auch die Wiedereingliederung in die Berufs- und Arbeitswelt nach der Transplantation. Den Willen zur Wiederaufnahme der Arbeit und Berufstätigkeit - vor der HT zum Ausdruck gebracht - haben wir u. a. als Prädiktor untersucht und gewichtet. Um die Gewichtung der verschiedenen psychosozialen Prädiktoren und Kriterien aus einer Reihe von Variablen geht es, neben ihrer inhaltlichen Darstellung, in dieser Arbeit. Soweit bekannt, hat sich bisher kein Autor an der Aufstellung einer Rangfolge für Prädiktoren und Kriterien versucht.

\section{Fragestellung und Ziel der Untersuchung}

Ausgehend von der Beobachtung, daß die Patienten, die seit 1981 in der Herzchirurgischen Klink des Klinikums Großhadern transplantiert werden, sehr unterschiedliche Befunde und Ausprägungen in somatischen wie psychosozialen Variablen aufwiesen, stellten wir uns, aufgrund bereits bekannter und eigens aufgestellter Parameter, folgende Fragen:

1. Welche Variablen erweisen sich als gute Prädiktoren im Hinblick auf die Rehabilitationsphase nach einer Herztransplantation?

2. Läßt sich eine Rangordnung der besten und universellsten Prädiktoren erstellen?

3. Welche Kriterien lassen sich aus den gefundenen Prädiktoren besonders gut vorhersagen?

\section{Patientengut und Methodik}

Die Untersuchungen fanden zwischen März 1986 und März 1991 in der Herzchirurgischen Abteilung des Klinikums Großhadern der LudwigMaximilians-Universität München statt. Die untersuchte Stichprobe bestand aus 44 Patienten, die sich einer Herztransplantation unterzogen, wovon 2 Patienten prä- und 6 Patienten postoperativ starben; ein Patient wurde wegen eines bestehenden $\mathrm{Al}$ koholismus abgelehnt.

Geschlecht: 6 Frauen und 38 Männer.

Altersverteilung: Minimum bei 18 Jahren, Maximum bei 56 Jahren, bei einem Durchschnittsalter von 42 Jahren und einer Standardabweichung von 10 .
Familienstand: 12 Patienten waren ledig, 27 verheiratet, 4 geschieden und einer verwitwet. Zur weiteren statistischen Analyse wurden die Geschiedenen und Verwitweten den Ledigen zugeschlagen, somit besteht die Stichprobe aus 27 verheirateten und 17 nicht verheirateten Patienten. Schulbildung: 28 Patienten hatten Grundschulabschluß, 10 eine Realschule besucht und 6 einen höheren Schulabschluß.

Diagnose: 31 Patienten litten an einer dilatativen Cardiomyopathie (DCM), 9 an einer coronaren Herzerkrankung (KHK), einer an einer neurogenen Myopathie und 3 an angeborenen Herzfehlern.

Schweregrad der Herzerkrankung (NYHA): 1 Patient hatte Beschwerden nur bei schwerer Belastung, 19 bei leichter Belastung und 25 schon im Ruhezustand.

Erkrankungsdauer: Sie betrug im Mittel 51/2 Jahre bei einer Standardabweichung von 5,63 mit Minimum bei nahezu 0 , Maximum bei $25 \mathrm{Jah}$ ren.

Körperlicher Zustand: Ihren präoperativen körperlichen Zustand bezeichneten 27 Patienten als schlecht, 10 als befriedigend und 7 als gut.

Motivation: 26 Patienten wurden als eindeutig motiviert eingestuft, 18 als ambivalent.

Religiosität: 17 Patienten bezeichneten sich als religiös, 27 als nicht religiös. Auf die Frage, ob sie Halt im Glauben fänden, antworteten $25 \mathrm{mit}$ ,ja“" und 19 mit „nein“.

Die Daten und Befunderhebungen stützen sich auf einen eigens für Interviews mit Herztransplantierten entwickelten Fragenkatalog, in dem alle für diese Untersuchung relevant erscheinenden Informationen gesammelt wurden.

Präoperativ wurden die Untersuchungen in Form eines semistrukturierten Interviews durchgeführt. Bei jeder Untersuchung wurde ein psychopathologischer Befund in Anlehnung an den Dokumentationsbogen 
des AMDP-Systems (Arbeitsgemeinschaft für Methode und Dokumentation in der Psychiatrie) erhoben. Bei der Krankheitsanamnese wurde besonderes Gewicht auf die psychologische Verarbeitung der Herzerkrankung und die bevorstehende Transplantation sowie auf die Motivation gelegt. Postoperativ wurden die Patienten, zunächst auf der Intensivstation, danach auf der Allgemeinstation, durchschnittlich $2 \times$ in der Woche untersucht. Nach der Entlassung aus der Klinik erfolgten weitere Untersuchungen nach 3, 6 und 12 Monaten. Bei 15 Patienten wurden über 1 Jahr hinaus, bis zu maximal 3 Jahren ( 7 Patienten) weitere Untersuchungen durchgeführt (Tabelle 1).

Die den statistischen Verfahren zugrunde liegenden 47 operationalisierten Variablen sind folgende:

Präoperative Variablen: 1. Schweregrad der Herzerkrankung nach NYHA, 2. Alter, 3. Geschlecht, 4. Diagnose, 5. Familienstand, 6. Wohnsituation, 7. Kinder, 8. Entfernung Wohnung - Klinik, 9. Schulbildung, 10. Berufsperspektive, 11. Er- krankungsdauer in Jahren, 12. Wartezeit insgesamt, 13. Wartezeit in der Klinik, 14. körperlicher Zustand vor der Transplantation (subjektiv), 15. länger bestehende Bettlägrigkeit, 16 . Religiosität, 17. Halt im Glauben, 18. Alkoholanamnese, 19. Beistand und Geborgenheit durch die Familie bzw. Partner, 20. Besuchshäufigkeit pro Woche, 21. Belastbarkeit der Partnerschaft durch die Erkrankung, 22. Dominanz in der Partnerschaft, 23. soziale Kontakte, 24. wichtigste Bezugsperson, 25. Mutter gestorben, 26. Vater gestorben, 27. Fernsehkonsum, 28. Kontakt zu anderen HTPatienten präoperativ, 29. Krankheitsverarbeitung, 30. HT-Verarbeitung, 31. Motivation, 32. sozialer Rückhalt, 33. Lebenszufriedenheit, 34. psychopathologischer Befund. Variablen Intensivstation: 35 . psychopathologischer Befund, 36. körperlicher Zustand.

Variablen postoperativer stationärer Aufenthalt: 37. psychopathologischer Befund, 38. körperlicher Zustand, 39. Besuchshäufigkeit pro Woche, 40. Beistand und Geborgenheit durch Familie bzw. Partner.

Tab. 1

\begin{tabular}{|c|c|c|c|}
\hline Zeitverlauf der Transplantationen & Mittelwert & Minimum & Maximum \\
\hline $\begin{array}{l}\text { Zeit zwischen Erstkontakt } \\
\text { und Transplantation }\end{array}$ & 1,8 Monate & 4 Tage & 15 Monate \\
\hline Wartezeit auf die Transplantation insgesamt & 5,3 Monate & 10 Tage & 22 Monate \\
\hline $\begin{array}{l}\text { Wartezeit auf die Transplantation in der } \\
\text { Klinik }\end{array}$ & 2,4 Monate & 6 Tage & 10 Monate \\
\hline Aufenthalt auf der Intensivstation $\left(^{*}\right)$ & 1,5 Wochen & 3 Tage & 5 Wochen \\
\hline Aufenthalt auf der Allgemeinstation $\left({ }^{* *}\right)$ & 3,7 Wochen & 10 Tage & 8 Wochen \\
\hline $\begin{array}{l}\text { Zeit zwischen Transplantation und } \\
\text { 1. Entlassungsuntersuchung }\end{array}$ & 3,1 Monate & 10 Wochen & 4 Monate \\
\hline $\begin{array}{l}\text { Zeit zwischen Transplantation und } \\
\text { 2. Entlassungsuntersuchung }\end{array}$ & 6,6 Monate & 5 Monate & 8 Monate \\
\hline $\begin{array}{l}\text { Zeit zw. Transplantat. und letzter } \\
\text { Untersuchung }\end{array}$ & 16,7 Monate & 12 Monate & 3 Jahre \\
\hline \multicolumn{4}{|c|}{$\begin{array}{l}\text { *: Werte exklusiv der } 2 \text { Patienten, die wegen Abstoßung bzw. Infekt } 9 \text { bzw. } 13 \text { Wochen } \\
\text { auf der Intensivstation verweilten } \\
\text { **: Werte exklusiv der Gestorbenen ( } 2 \text { Patienten unmittelbar nach der Operation, } 2 \text { in- } \\
\text { nerhalb der } 1 \text {., einer in der } 2 \text {. und } 1 \text { Patient in der } 4 \text {. Woche postoperativ) }\end{array}$} \\
\hline
\end{tabular}

Variablen nach einem Jahr (gleichzeitig Kriterien für die Prognose. Siehe Tabelle 2): 41. psychopathologischer Befund, 42. körperlicher Zustand, 43. körperliche Belastbarkeit, 44. soziale Reintegration, 45 . berufliche Rehabilitation, 46. Lebenszufriedenheit, 47. Compliance.

\section{Statistische Verfahren}

Aufgrund von skalentheoretischen und Verteilungsüberlegungen kamen für die meisten quantitativen statistischen Analysen nonparametrische Verfahren zur Anwendung. Die Verfahren sind im einzelnen folgende: 1 . Der Chi'-Test, 2. Friedman's Varianzanalyse, 3. der Mann-Whitney-UTest, 4. Kruskal-Wallis-H-Test. Alpha-Risiko bei allen Tests $=5 \%$.

Die Variablen wurden nach der regressionsanalytischen Terminologie zuerst in 2 Klassen eingeteilt:

a) Kriterien (vorherzubestimmende Variablen)

b) Prädiktoren (Vorhersagevariablen).

Als Kriterien für die Prognose 1 Jahr postoperativ wurden untersucht:

1. Die Compliance, 2. der körperliche Zustand, 3. die soziale Reintegration, 4 . die berufliche Rehabilitation, 5. die körperliche Belastbarkeit, 6. der psychopathologische Befund, 7. die Lebenszufriedenheit.

\section{Ergebnisse}

Die statistische Auswertung umfaßte 3 Dinge:

1. Korrelation der Kriterien

2. Bestimmung der Prädiktoren

3. Erstellung einer Rangordnung der Prädiktoren und Kriterien. 


\section{Korrelation der Kriterien}

Im ersten Schritt wurde versucht, mögliche Zusammenhänge zwischen diesen vorherzusagenden Kriterien aufzudecken. Hierzu wurden $\mathrm{Chi}^{2}$ Tests anhand von Kreuztabellierungen durchgeführt. Gegenübergestellt wurden jedem möglichen medizinisch-somatischen Kriterium (Compliance, körperlicher Zustand, körperliche Belastbarkeit) die soziologisch-psychologischen Variablen (soziale Reintegration, berufliche Rehabilitation, psychopathologischer Befund, postoperative Lebenszufriedenheit). Es ergaben sich folgende si- gnifikante Zusammenhänge, die sich kurz in ,je-desto"-Aussagen zusammenfassen lassen:

Je besser die Compliance, desto:

- unauffälliger der psychopathologische Befund,

- besser die soziale Reintegration

- besser die berufliche Rehabilitation

- höher die Lebenszufriedenheit.

Je besser der körperliche Zustand, desto:

- besser die soziale Reintegration.

Je höher die körperliche Belastbarkeit, desto:

- besser die soziale Reintegration.

$\mathrm{Da}$ es sich hier um Zusammenhangaussagen handelt, d. h. es wurde im
Vorhinein nicht bestimmt, was Ursache und was Wirkung ist, können die Aussagen auch invers formuliert werden; für jedes „Desto“ kann also auch ein "Je" gesetzt werden und vice versa.

\section{Prädiktoren für die 7 Kriterien}

In einem zweiten Schritt wurden die signifikanten Prädiktoren für die 7 Kriterien bestimmt.

Zur statistischen Analyse wurden zunächst mit Hilfe einer Serie von Mann-Whitney-U-Tests, deren Teststärke mit $95 \%$ nahe an die der parametrischen $\mathrm{t}$-Tests herankommt, an-

Tab. 2

\begin{tabular}{|c|c|c|c|c|c|c|c|c|c|c|}
\hline \multicolumn{2}{|c|}{ Hierarchie der Prädiktoren und Kriterien } & \multicolumn{9}{|c|}{ Kriterien (1 Jahr postoperativ) } \\
\hline Prädikatoren (präoperativ) & nähere Bestimmung & 1 & 2 & 3 & 4 & 5 & 6 & 7 & $\Sigma$ & Rang \\
\hline $\begin{array}{l}\text { Motivation } \\
\text { psychopatholog. Befund }\end{array}$ & $\begin{array}{l}\text { eindeutig } \\
\text { unauffällig }\end{array}$ & * & $\begin{array}{l}* \\
*\end{array}$ & * & $\begin{array}{l}* \\
*\end{array}$ & * & $\begin{array}{l}* \\
*\end{array}$ & & $\begin{array}{l}6 \\
6\end{array}$ & 1 \\
\hline $\begin{array}{l}\text { sozialer Rückhalt } \\
\text { Berufsperspektive }\end{array}$ & $\begin{array}{l}\text { befriedigend - gut } \\
\text { ja }\end{array}$ & * & * & * & * & * & * & * & $\begin{array}{l}5 \\
5\end{array}$ & 2 \\
\hline $\begin{array}{l}\text { Geborgenh. durch Fam. } \\
\text { Krankheitsverarbeitung } \\
\text { HT-Verarbeitung }\end{array}$ & $\begin{array}{l}\text { viel } \\
\text { reif } \\
\text { reif }\end{array}$ & $\begin{array}{l}* \\
* \\
*\end{array}$ & $\begin{array}{l}* \\
* \\
*\end{array}$ & * & * & * & & & $\begin{array}{l}4 \\
4 \\
4\end{array}$ & 3 \\
\hline $\begin{array}{l}\text { Halt im Glauben } \\
\text { soziale Kontakte } \\
\text { Schweregrad (NYHA) } \\
\text { Bettlägerigkeit } \\
\end{array}$ & $\begin{array}{l}\text { ja } \\
\text { häufig } \\
\text { IV (im Ruhezustand) } \\
\text { ja }\end{array}$ & $\begin{array}{l}* \\
* \\
* \\
*\end{array}$ & $\begin{array}{l}* \\
* \\
* \\
*\end{array}$ & * & * & * & & & $\begin{array}{l}3 \\
3 \\
3 \\
3\end{array}$ & 4 \\
\hline $\begin{array}{l}\text { Wesensänderg. wegen } \mathrm{HK} \\
\text { Lebenszufriedenheit } \\
\text { Beistand (stationär) } \\
\text { Fernsehkonsum } \\
\end{array}$ & $\begin{array}{l}\text { geduldig, ruhiger } \\
\text { schlecht } \\
\text { viel } \\
\text { weniger als } 4 \text { Abende } \\
\end{array}$ & * & * & * & $\begin{array}{l}* \\
* \\
* \\
\end{array}$ & * & & * & $\begin{array}{l}2 \\
2 \\
2 \\
2 \\
\end{array}$ & 5 \\
\hline $\begin{array}{l}\text { Kinder } \\
\text { wichtigste Kontaktperson } \\
\text { Partnerdominanz } \\
\text { Mutter noch lebend } \\
\text { Vater noch lebend } \\
\text { belastete Beziehung } \\
\text { Wohnsituation } \\
\text { Alkoholanamnese } \\
\text { Geschlecht } \\
\text { Temperament }\end{array}$ & $\begin{array}{l}\text { ja } \\
\text { Partner } \\
\text { Patient selbst } \\
\text { ja } \\
\text { ja } \\
\text { nein } \\
\text { zusammen } \\
\text { negativ } \\
\text { weiblich } \\
\text { viel }\end{array}$ & $\begin{array}{l}* \\
* \\
*\end{array}$ & & * & & * & * & * & $\begin{array}{l}1 \\
1 \\
1 \\
1 \\
1 \\
1 \\
1 \\
1 \\
1 \\
1 \\
\end{array}$ & 6 \\
\hline \multicolumn{2}{|c|}{ Spaltensumme der Signifikanzen $\left({ }^{*}\right.$ bei $\leq .0 .5$ ) } & 14 & 12 & 10 & 10 & 8 & 5 & 5 & 64 & \\
\hline \multicolumn{2}{|c|}{ Rangstufe bezüglich der Vorhersagequalität } & 1. & 2. & 3. & & 4. & 5. & $\mathrm{Ra}$ & & \\
\hline
\end{tabular}

Schlüssel der Kriterien:

1 = soziale Reintegration

2 = Lebenszufriedenheit

$3=$ Compliance

$4=$ psychopathologischer Befund

$5=$ berufliche Rehabilitation

$6=$ körperliche Belastbarkeit

$7=$ körperlicher Zustand 
hand aller präoperativen dichotomen oder dichotomisierten Variablen Gruppen gebildet und die errechneten Kriterien-Mediane miteinander verglichen und deren Unterschiede auf Signifikanz geprüft. Danach wurden Kruskal-Wallis-Tests für die präoperativen Variablen mit mehr als zwei Ausprägungen durchgeführt.

\section{Rangordnung der Prädiktoren und Kriterien}

In einem dritten Schritt wurde eine Rangordnung für Prädiktoren und Kriterien aufgestellt. Dazu mußte die Voraussagequalität operationalisiert werden. Hierzu wurde zeilenweise für jeden Prädiktor die Zahl der Signifikanzen aus Tabelle 2 summiert und anschließend hierarchisiert. Aus den insgesamt 64 Signifikanzen ergibt sich folgende

Rangordnung der Prädiktoren

1. Eindeutige Motivation und unauffälliger psychopathologischer Eingangsbefund (je 6 Sterne von 7 möglichen)

2. Guter sozialer Rückhalt und die Absicht, in das Berufsleben zurückzukehren (je 5 Sterne)

3. Geborgenheit in der Familie, reife Krankheits- und HT-Verarbeitung (je 4 Sterne)

4. Bettlägrigkeit, Halt im Glauben, häufige Sozialkontakte (je 3 Sterne) sind allgemeinere und bereichsübergreifende Prädiktoren

5. Ein hoher Schweregrad der Krankheit, mangelhafte präoperative Lebenszufriedenheit, wenig Fernsehkonsum, Wesensänderung durch die Krankheit in Richtung "geduldiger, ruhiger", viel Beistand während des stationären Aufenthaltes (je 2 Sterne)

6. Weibliches Geschlecht, mindestens ein Elternteil lebend, fehlende Alkoholanamnese, Partner als wichtigste Kontaktperson, Dominanz des Patienten in der
Partnerschaft, keine Belastung der Beziehung durch die Krankheit, viel Temperament, die Wohnsituation mit Familie und Kindern (je 1 Stern) sind bereichsspezifische Prädiktoren

Keine Signifikanz ergab sich bei den Variablen: Geschwisterkonstellation, Religiosität, Verschlechterung des präoperativen Zustands, Ehestand, präoperative Diagnose, Entfernung der Wohnung von der Klinik, Schulbildung, präoperativer körperlicher Zustand, Angstprobleme, Kontakt zu anderen HT-Patienten. Sie kommen somit als valide Prädiktoren weniger in Frage.

$\mathrm{Zu}$ einer Rangordnung der am besten vorhersagbaren Kriterien kommt man, indem man die Spaltensummen der 25 signifikanten Prädiktoren in Tabelle 2 für jedes einzelne Kriterium addiert. Es entstand folgende

Rangordnung der Kriterien

1. Die soziale Reintegration (14 Sterne von 25 möglichen)

2. Die postoperative Lebenszufriedenheit (12 Sterne)

3. Die Compliance

Der psychopathologische Befund (je 10 Sterne)

4. Die berufliche Rehabilitation ( 8 Sterne)

5. Die körperliche Belastbarkeit Der körperliche Zustand (je 5 Sterne)

Die 7 Kriterien werden im folgenden, ihrer Rangordnung entsprechend, jetzt näher beschrieben und auf die für sie signifikanten Prädiktoren wird noch einmal kurz eingegangen.

\section{Die soziale Reintegration}

Die Wiedereingliederung in die Familie und in die Gesellschaft nach einer Herztransplantation sollte nach einer Zeit der Anpassung ohne wesentliche Veränderung in der Lebensführung geschehen. Der seit Be- ginn der Erkrankung in die Patientenrolle gedrängte Herzempfänger hat im Idealfall seine früheren Aktivitäten aufgenommen und hat seine Aufgaben und Pflichten besonders in der Familie zum Abbau einer möglichen Überlastung einzelner Familienmitglieder wieder übernommen. $29(83 \%)$ Patienten waren $1 \mathrm{Jahr}$ nach der Herztransplantation gut, 4 $(11 \%)$ befriedigend und $2(6 \%)$ schlecht reintegriert.

Für das Kriterium „soziale Reintegration" wurden 14 signifikante Prädiktorvariablen ermittelt, wobei die Prädiktoren in der Reihenfolge ihrer Signifikanzladungen (von maximal 6 bis minimal 1), d.h. ihrer Rangordnung nach, aufgeführt werden (Tabelle 2, Kriterium 1).

Demnach läßt sich sagen: Wer nach einem bzw. drei Jahren sozial gut reintegriert war, war präoperativ psychisch unauffällig und eindeutig motiviert, hatte die Herzerkrankung und die bevorstehende Transplantation reif und adäquat verarbeitet, war präoperativ in einer schlechten Allgemeinverfassung, kam aus einem überwiegend guten sozialen Umfeld mit intakter Beziehung und hatte Geborgenheit in der Familie und viele soziale Kontakte, wohnte nicht allein und fand Halt in seinem Glauben.

\section{Die postoperative \\ Lebenszufriedenheit}

Die Lebenszufriedenheit ist ein übergeordneter Begriff für sehr unterschiedliche Kategorien. Die Patienten wurden aufgefordert, eine allgemeine Beurteilung $\mathrm{zu}$ folgenden Bereichen zu geben: Ehe/Partnerschaft, Familie, Sexualleben, soziale Kontakte, berufliche Situation, Wohnsituation, Freizeitgestaltung, Zufriedenheit mit sich selbst. Vorgegebene Wertskala: 1 (sehr gut), bis 6 (sehr schlecht). Die einzelnen Punkte gingen als Summe in die Beurteilung 
gut $(23$ Pat. $=66 \%)$, befriedigend $(7=20 \%)$ und schlecht $(5=14 \%)$ ein.

Es wurden 12 signifikante Prädiktorvariablen für die postoperative Lebenszufriedenheit ermittelt (Tabelle 2, Kriterium 2).

Die besten Voraussetzungen für eine gute postoperative Lebenszufriedenheit haben demnach präoperativ psychopathologisch unauffällige und eindeutig motivierte Patienten mit reifer Krankheits- und HTVerarbeitung, mit präoperativ weit fortgeschrittenem Krankheitsbild und einem guten sozialen Rückhalt, die vor der Transplantation bereits eine spätere berufliche Perspektive haben.

\section{Die Compliance}

Compliance ist in dieser Untersuchung definiert als Bereitschaft des Patienten, bei diagnostischen und therapeutischen Maßnahmen mitzuwirken. Hierzu gehören absolute Zuverlässigkeit bezüglich der Medikamenteneinnahme, genaues Einhalten der Diätvorschriften, Alkohol- und Nikotinabstinenz, Beachtung allgemeiner Verhaltensregeln zur Infektionsprophylaxe, Einhaltung allgemeiner Kontrolltermine bei Hausärzten und Chirurgen, sofortige Kontaktaufnahme mit den Ärzten bei Verschlechterung des Befindens und die Bereitschaft, auch bei guter Gesundheit in regelmäßigen Abständen aufwendige und intensive Untersuchungen auf sich zu nehmen. Nach dieser Definition wurde eine Einteilung in "gut", „befriedigend“ und "schlecht" vorgenommen. Von den 35 Patienten zeigten $22(63 \%)$ eine gute Compliance, d.h., alle oben beschriebenen Punkte wurden befolgt. $9(26 \%)$ hatten eine befriedigende Compliance und 4 Patienten $(11 \%)$ zeigten schwere Compliancestörungen, d. h. ein Verweigern der Kooperation in mehreren bzw. in allen Punkten.
Im einzelnen konnten 10 Prädiktorvariablen für das Kriterium Compliance ermittelt werden (Tabelle 2, Kriterium 3).

Zusammenfassend kann gesagt werden, daß die Patienten, die postoperativ eine gute Compliance aufwiesen, präoperativ psychopathologisch unauffällig und eindeutig motiviert waren, eine reife Krankheitsund Herztransplantationsverarbeitung bei eher schlechtem Allgemeinzustand aufwiesen, von einem guten sozialen Umfeld mit Kindern und viel familiärer Geborgenheit umgeben waren, sich vom Wesen her eher ruhiger und sensibler zeigten und Halt im Glauben fanden. Andere Variablen, wie Alkoholanamnese, soziale Kontakte und Ehestand zeigten zwar Unterschiede bei den Compliancewerten, ergaben aber keinen signifikanten Zusammenhang.

\section{Der psychopathologische Befund}

Psychische Auffälligkeiten und eine gestörte psychosoziale Anpassung wurden im psychopathologischen Befund erfaßt. Dieser ist neben dem somatischen Befund ein Hauptkriterium für die Beurteilung der Patienten. Von den 35 Patienten waren 24 $(68 \%)$ unauffällig, $7(20 \%)$ leicht und $4(12 \%)$ schwer auffällig. Im folgenden wird zunächst eine inhaltliche Beschreibung dieser Einteilung vorgenommen:

a) Psychopathologisch unauffällige Patienten waren fähig, die Herztransplantation psychisch normal zu bewältigen. Dazu zählte auch, daß die normalpsychologischen begründeten Befürchtungen und Ängste vor der Transplantation und vor den möglichen Komplikationen nach der Transplantation offen zur Sprache gebracht oder auf Befragen sofort zugegeben wurden, ohne daß diese jedoch Krankheitswert bekamen, also zur Symptomatik wurden. b) Bei psychopathologisch leicht auffälligen Patienten fand sich eine deutliche Neigung zu Depression, Angst, Aggression, Dysphorie, Apathie, Mißtrauen, Stimmungsschwankungen oder auch zu Verleugnung von Ängsten. Auch situationinadäquate euphorische bis leicht manische Verstimmungen und leichtere psychoorganische Syndrome zählten dazu.

c) Als schwer auffällig wurden $\mathrm{Pa}$ tienten bezeichnet, bei denen die oben aufgezählten Symptome einen sehr starken Ausprägungsgrad erreichten.

Für einen postoperativ psychopathologisch unauffälligen Befund ergaben sich 10 Prädiktorvariablen (Tabelle 2, Kriterium 4).

Demnach sind die präoperativ psychopathologisch unauffalligen Patienten in der Regel auch hinterher wieder unauffällig. So waren in dieser Studie auch alle ursprünglich $16(46 \%)$ der vor der HT unauffälligen Patienten ein Jahr nach der Transplantation wieder unauffällig. Sie waren auch eindeutig motiviert, hatten einen guten sozialen Rückhalt und hatten ihre Krankheit und die bevorstehende Transplantation psychologisch reif verarbeitet. Die Krankheit hatte bei ihnen eine Wesensänderung in Richtung zu mehr Ruhe und Geduld bewirkt, und es bestand schließlich bei ihnen der Wunsch, nach der Transplantation ihre berufliche Tätigkeit wieder aufzunehmen.

\section{Die berufliche Rehabilitation}

Die berufliche Rehabilitation gilt als geglückt, wenn die Patienten $1 \mathrm{Jahr}$ nach der Transplantation vollständig wieder in ihrem alten oder einem neuen Beruf beschäftigt sind und keine Rente beziehen. Dies war bei $20(57 \%)$ Patienten der Fall. Hierzu zählen auch die Hausfrauen bzw. Männer, die die vor der Erkrankung 
geleistete Arbeit wieder aufnahmen. $2(6 \%)$, waren ,befriedigend" reintegriert. Sie konnten ihre Tätigkeit nur teil- oder zeitweise ausüben, und 13 $(37 \%)$ waren nicht beruflich reintegriert. Sie waren dauerhaft berentet, nicht arbeitswillig oder zu alt, um in den Beruf zurückzukehren. Es ließen sich 8 Prädiktorvariablen für eine geglückte berufliche Rehabilitation feststellen (Tabelle 2, Kriterium 5).

Wie erwartet hatten Patienten mit einer präoperativen beruflichen Perspektive eine bessere berufliche Rehabilitation. Frauen gelingt es leichter, ihre ehemalige Tätigkeit wieder aufzunehmen (was allerdings nicht gleichbedeutend damit ist, in eine Berufstätigkeit zurückzufinden). Eine eindeutige Motivation und ein unauffälliger psychopathologischer Befund und ein sozialer Rückhalt und Beistand und Geborgenheit durch den Partner oder die Familie, sowie präoperative Unzufriedenheit mit dem Leben (schlechte Lebensqualität!) bei hohem Erkrankungsgrad verbesserten die berufliche Wiedereingliederung.

\section{Die körperliche Belastbarkeit}

Die Patienten wurden aufgefordert, ihre körperliche Belastbarkeit im Vergleich zur Zeit vor der Herzerkrankung einzuschätzen. Folgende Einteilungen wurden vorgegeben: wieder voll belastbar $(20$ Pat. $=$ $57 \%)$, nur bedingt belastbar $(14=$ $40 \%$ ), wenig bis gar nicht belastbar $(1=3 \%)$. Es ergaben sich 5 Prädiktorvariablen (Tabelle 2, Kriterium 6).

Das heißt mit anderen Worten: Wer schon vor der Transplantation Perspektiven für die Zukunft hatte, insbesondere berufliche, eindeutig motiviert und psychopathologisch unauffällig war, der schätzte sich ein Jahr nach der Transplantation auch wieder als voll belastbar ein. Überraschend erwiesen sich auch noch lebende Eltern als positiv für den $\mathrm{Pa}$ - tienten. Lebende Eltern bedeuten demnach für schwer Kranke eine Zukunftsperspektive.

\section{Der körperliche Zustand}

Bei der Beurteilung des körperlichen Zustandes wurde das gegenwärtige allgemeine körperliche Wohlbefinden eingeteilt in "gut" (28 Patienten $=80 \%)$, ,befriedigend" $(6 \mathrm{~Pa}$ tienten $=17 \%)$ und ,schlecht" $(1 \mathrm{~Pa}$ tient $=3 \%$ ). Hierbei wurden sowohl subjektive Kriterien (Selbsteinschätzung des Patienten, noch bestehendes Krankheitsgefühl) als auch objektive Kriterien (Gesundheitszustand aus ärztlicher Sicht, Komplikationen, Abstoßungsreaktionen etc.) berücksichtigt.

Für einen guten körperlichen $\mathrm{Zu}$ stand nach einem Jahr ergaben sich ebenfalls 5 Prädiktorvariablen (Tab. 2, Kriterium 7).

Es zeigte sich demnach, daß die signifikanten Prädiktoren für einen guten körperlichen Zustand ein Jahr nach der Transplantation das Vorhandensein beruflicher Perspektive, eine schlechte präoperative Lebenszufriedenheit, Dominanz in der Partnerschaft, eine Wesensart mit viel Temperament und eine negative Alkoholanamnese waren. Der Schweregrad der Herzerkrankung nach der NYHA und die Diagnose der Herzerkrankung hatten keinen Einfluß auf den postoperativen körperlichen Zustand.

\section{Diskussion}

Ziel der Prädiktorforschung ist es, Faktoren zu bestimmen, die einen Therapieerfolg voraussagen. Dieser Therapieerfolg bzw. Mißerfolg bei Herztransplantierten konnte in einer früheren Prädiktorstudie - gemessen an der postoperativen Lebensqualität und an der Compliance - z. B. aufgrund des Vorhandenseins oder
Nichtvorhandenseins einer schweren Persönlichkeitsstörung vorausgesagt werden (1). In einer anderen Studie kam man zu dem Ergebnis - gemessen an der Vulnerabilität durch Stress' nach der Herztransplantation -, daß eine psychiatrische Anamnese, jüngeres Alter, Erleiden schwerer Verluste, geringes Selbstbewußtsein und die Unfähigkeit, mit Problemen, insbesondere mit Gesundheitsproblemen, fertigzuwerden, eine ungünstige Prognose für den Therapieerfolg darstellen (4). In einer dritten Prädiktorstudie wurde ein positiver klinischer Erfolg der Herztransplantation vorausgesagt, wenn die psychosozialen Variablen Empathie, Fürsorge und Unterstützung vonseiten des Lebenspartners, wenig Anforderungen hinsichtlich gefühlsgebundener Kommunikation, Genauigkeit, Belastbarkeit, emotionale Beherrschtheit, hohe Frustrationstoleranz, niedrige Aggressionsschwelle und niedrigeres Lebensalter vorlagen (2), Merkmale, die wir im wesentlichen auch in unserer Studie, wenn auch $z$. T. unter anderen Begriffen und Kategorien, erfaßt haben und die wir auch als signifikante Prädiktoren nachweisen konnten.

In keiner der drei genannten Studien wurde die Motivation, die bereits in den Anfängen der Herztransplantation als ein wichtiger Prädiktor für den Erfolg der Transplantation galt, als Variable mituntersucht (9) und in keiner der uns bislang bekannten Studien überhaupt, die die psychosozialen Bedingungen der Herztransplantation untersuchen, hat man sich mit der Rangfolge bzw. Wertigkeit von Prädiktoren befaßt. Aufgrund unserer Studie konnten wir eine sechsstufige Rangfolge für soziale, psychische und körperliche Prädiktorvariablen aufstellen, und neben der Prädiktorrangordnung auch eine Rangordnung der Kriterien für den Therapieerfolg ermitteln.

Die besten Prädiktoren für eine erfolgreiche Transplantation sind dem- 
nach (Rang 1) eine eindeutige Motivation und ein unauffälliger präoperativer psychopathologischer Befund, gefolgt von (Rang 2) einem guten sozialen Rückhalt und einer weiterbestehenden Berufsperspektive. Es folgen (Rang 3) die reife psychologische Verarbeitung der Herzerkrankung und der bevorstehenden Transplantation und das Gefühl der Geborgenheit durch die Familie. Danach sind (Rang 4) ein fortgeschrittener Krankheitszustand, insbesondere der Umstand einer bereits eingetretenen Bettlägrigkeit, häufig bestehende Sozialkontakte und Halt im Glauben drei weitere wichtige Prädiktoren. Prognostisch günstig sind ferner (Rang 5) eine im Verlaufe der Herzerkrankung eingetretene „Wesensänderung" des Patienten in Richtung einer geduldigen und ruhigen Wesensart, ein starker familiärer Beistand während des stationären Aufenthaltes, Fernsehkonsum von weniger als an 4 Abenden und die Einstufung der Lebenszufriedenheit („Lebensqualität"!) mit „schlecht". Von Bedeutung ist schließlich, jedoch am Schlu $\beta$ rangierend (Rang 6), daß die Beziehung zum Partner als die wichtigste Beziehung zu einer Kontaktperson überhaupt gilt, daß sie nicht belastet ist und da $\mathrm{B}$ eine gemeinschaftliche Wohnsituation besteht. Von Vorteil ist auch, wenn der Patient selbst der dominierende Teil ist, und auch, wenn beide Eltern des Patienten noch leben und wenn Kinder da sind. Frauen haben eine bessere Prognose als Männer, und wer sich durch viel Temperament auszeichnet und keinen oder nur wenig Alkohol trinkt, ist ebenfalls im Vorteil.

Keine Signifikanz ergab sich bei den Variablen Geschwisterkonstellation, Religiosität, Verschlechterung des präoperativen Zustands, Ehestand, präoperative Diagnose, Entfernung der Wohnung von der Klinik, Schulbildung, präoperativer körperlicher Zustand, Angstpro- bleme und Kontakt zu anderen HTPatienten. Als valide Prädiktoren kommen diese somit weniger in Frage.

Die Rangordnung der Kriterien, die ermittelt wurde, ergab, daß an erster Stelle (Rang 1) die soziale Integration steht, gefolgt von (Rang 2) der postoperativen Lebenszufriedenheit. Danach kommt mit gleicher Wertigkeit (Rang 3) die Compliance und der psychopathologische Befund, danach (Rang 4) die berufliche Rehabilitation. An letzter Stelle (Rang 5) stehen gemeinsam die körperliche Belastbarkeit und der körperliche Zustand.

Die Rangordnung der Prädiktoren zeigt, daß neben der eindeutigen Motivation - d. h. AusschluB jeglicher Ambivalenz -, der unauffällige präoperative psychopathologische Befund mit an erster Stelle rangiert. Psychopathologisch unauffällig bedeutet nicht Abwesenheit von natürlicher, normalpsychologischer Angst (Realangst), die mit zum Bewältigungsproze $\beta$ des Transplantationsschicksals gehört und deren Verdrängung oder Verleugnung in der Wartezeit als psychische Auffälligkeit zu werten ist. Ein signifikanter Prädiktor war das Vorhandensein oder Nichtvorhandensein von Angst vor der Transplantation nicht, wohl aber die Variable der gelungenen oder nicht gelungenen psychologischen Verarbeitung der Herzerkrankung und der bevorstehenden Transplantation und das Gefühl und Bewußtsein der familiären Geborgenheit, die den sozialen Rückhalt und die Absicht, ins Berufsleben zurückzukehren, mit begründen und verständlich machen.

Neben häufigen Sozialkontakten, ein Merkmal für Extroversion, haben wir, unseres Wissens erstmals, auch den Halt im (kirchlichen) Glauben als einen Prädiktor herausgearbeitet. Die bloße Zugehörigkeit zur Religionsgemeinschaft oder Konfession dagegen ist kein signifikanter
Prädiktor, ebensowenig wie es das formale Kriterium des Ehestandes, ohne die entsprechende innere Bindung zum Ehepartner, ist.

Von den krankheitsbezogenen Prädiktoren ist die Bettlägrigkeit ein noch härteres Kriterium als die bloße Zuordnung zum klinischen Schweregrad IV NYHA. Beides ist keineswegs immer identisch. Es gibt eine Reihe von Patienten, die in bereits fortgeschrittenem Krankheitsstadium der Transplantation gegenüber immer noch ambivalent eingestellt bleiben, selbst wenn die Lebensqualität dabei sehr eingeschränkt ist. Erst die Erfahrung der Hilflosigkeit bis zur Bettlägrigkeit führt zu einem eindeutigen Entschluß zur Transplantation. Das steht auch im Einklang damit, daß die Variable „präoperativer körperlicher Zustand" und selbst die der „Verschlechterung des präoperativen Zustandes" keine Signifikanz aufweisen. Es verwundert, daß dies auch für die Variable „Kontakt zu anderen HT-Patienten" gilt, da der Wunsch, mit bereits Transplantierten Kontakt aufzunehmen, häufig geäuBert wird und im individuellen Fall sehr hilfreich sein kann.

In der Rangordnung der Kriterien für den Therapieerfolg steht die soziale Reintegration an erster Stelle. Das zeigt, daß für den Transplantierten die Tatsache und das Bewußtsein, in einer Gemeinschaft wieder integriert zu sein, höher einzuschätzen ist bzw. einen höheren Stellenwert einnimmt, als das körperliche und psychische Wohlbefinden. Wir sehen: die Herztransplantation ist in erster Linie das Geschenk eines neuen, zweiten Lebens, sie verschafft dem Menschen aber keine neue Psyche, in der das subjektive Wohlbefinden als höchstes Gut rangiert. Compliance und psychopathologischer Befund entsprechen sich. Wer compliancefähig ist, der zeigt, daß er das neu geschenkte Leben nicht aufs Spiel setzt, daß das Leben an sich be- 
reits das höchste Gut ist, und nicht eine möglichst hohe Lebensqualität. Das zeigt sich auch in der „Werteverschiebung" nach der Transplantation in bezug auf den Wunsch, Beruf und Arbeit wieder aufzunehmen. Als Prädiktor nimmt der Wunsch und Wille, in das Berufsleben zurückzukehren, als zweiter in der Rangfolge - nach der eindeutigen Motivation und dem unauffälligen psychischen Zustand - einen hohen Stellenwert ein. Er stärkt nicht nur die Motivation, sondern ist auch Ausdruck einer positiven Erwartungshaltung für die Zukunft, die bereits selbst ein Prädiktor für einen Transplantationserfolg ist $(2,8)$. Als Kriterium steht die berufliche Rehabilitation später an vorletzter Stelle, vor der körperlichen Belastbarkeit und dem körperlichen Zustand. Wenn dieser Rangabfall sicher auch etwas damit zu tun hat, daß es für den Transplantierten objektiv größere
Schwierigkeiten gibt, in den Beruf zurückzukehren, als er sich dies vorgestellt hatte $(5,11)$, so hat es aber auch damit zu tun, daß sich die Lebensphilosophie des Transplantierten in der Regel ändert: Die Sinnerfüllung des Lebens kreist nach der Transplantation nicht mehr in dem Maße um Arbeit und Beruf, wie dies zuvor der Fall war, als sie ihm durch die Krankheit genommen wurden.

Die Rangordnung der Kriterien entspricht auch einer Wertung. Die Lebenszufriedenheit, die nach der sozialen Integration an zweiter Stelle steht, ist ein sehr wichtiges Kriterium. Lebenszufriedenheit ist aber mehr als Lebensqualität im Sinne der bloßen Wiederherstellung von Funktionen. Die Definition des Therapieerfolges nach Herztransplantation ist in den letzten Jahren zum Problem geworden, weil der Begriff der Lebensqualität das eigentliche Kriterium des Therapieerfolges ge- worden ist $(3,6,10,12)$, das sogar die Frage nach den Überlebensraten. die früher ganz im Vordergrund stand, nicht nur ergänzte, sondern fast aus den Augen verloren hat. Die Frage: Wie lebe ich weiter? ist scheinbar wichtiger geworden, als die Frage: Wie lange lebe ich weiter? Das Geschenk eines neuen oder zweiten Lebens, das durch die Herztransplantation gewährt wird, wird nicht mehr als das empfunden, was es ursprünglich war, nämlich weiterlebenkönnen und -dürfen, in allererster Linie leben, und nicht selbstverständliche Anknüpfung an das normale Leben, wie es vor Eintritt der Herzerkrankung war, das heute Thema des wissenschaftlichen Interesses der Lebensqualitätsforschung ist. Es ist aber mehr zum Problem des Arztes und Wissenschaftlers, als zu dem des Transplantierten geworden, der weiß, vor der Lebensqualität kommt das Leben.

\section{Literatur}

1. Brennan AF, Davis MH, Buchholz DJ, Kuhn WF, Gray LA (1987) Predictors of quality of life following cardiac transplantation - Importance of compliance with medical regimen, especially immunosuppressant therapy. Psychosomatics 28: 566-571

2. Bunzel B, Wollenek G (1994) Heart Transplantation: are there Psychosocial Predictors for Clinical Success of Surgery? Thorac cardiovasc Surgeon 42: 103-107

3. Caine N, Sharples L, Wallwork L (1992) Quality of life before and after heart transplantation. In: Walter PJ (eds) Quality of life after open-heart surgery. Kluwer Dordrecht, pp 445-455

4. Dew MA, Simmons RG, Roth LH, Schulberg HC, Thompson ME, Armitage JM, Griffith BP (1994) Psychological predictors of vulnerability to distress in the year following heart transplantation. Psychol Med 24: 929-945
5. Duitsman DM, Cychosz ChM (1994) Psychosocial Similarities and Differences among Employed and Unemployed Heart Transplant Recipients. J Heart and Lung Transplant 13: 108-115

6. Erdman RH, Hostman L, van-Domburg RT, Meeter K, Balk AH (1993) Compliance with the medical regime and partner's quality of life after heart transplantation. Quality of Life Research 3: 204-212

7. Jones BM, Taylor F, Downs K, Spratt $P$ (1992) Longitudinal study of quality of life and psychological adjustment after cardiac transplantation. Med $J$ Australia 157: $24-26$

8. Leedham B, Meyerowitz BE, Muirhead J, Frist WH (1995) Positive expectations predict health after heart transplantation. Hlth Psychol 14: 74-79

9. Meyendorf R, Dassing $\mathbf{M}$, Scherer J, Klinner W, Kemkes B, Reichart B (1989) Prädiktive und rehabilitative Gesichts- punkte bei Herztransplantierten. Herz 14: $308-321$

10. Meyerowitz BE, Vasterling J, Muirhead J, Frist W (1990) Quality of life and coping in heart transplant recipients. In: Willner AE, Rodewald G (eds) Impact of Cardiac Surgery on the Quality of Life. Plenum Press, pp 471-481

11. Paris W, Woodbury A, Thompson S, Levick $M$, Nothegger $S$, Arbuckle $P$, Hutkin-Slade L, Cooper DK (1993) Returning to work after heart transplantation. J Heart and Lung Transplant 12: $46-53$

12. Rosenblum DS, Rosen ML, Pine ZM, Rosen SH, Borg-Stein J (1993) Health status and quality of life following cardiac transplantation. Arch Physical and Medical Rehabil 74: 490-493 